\title{
Depth-dependent EBIC microscopy of radial-junction Si micropillar arrays
}

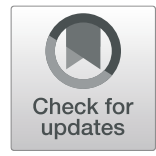

Kaden M. Powell ${ }^{1}$ and Heayoung P. Yoon ${ }^{1,2^{*}}$ (D)

\begin{abstract}
Recent advances in fabrication have enabled radial-junction architectures for cost-effective and high-performance optoelectronic devices. Unlike a planar PN junction, a radial-junction geometry maximizes the optical interaction in the three-dimensional (3D) structures, while effectively extracting the generated carriers via the conformal PN junction. In this paper, we report characterizations of radial PN junctions that consist of $p$-type Si micropillars created by deep reactive-ion etching (DRIE) and an $n$-type layer formed by phosphorus gas diffusion. We use electron-beam induced current (EBIC) microscopy to access the 3D junction profile from the sidewall of the pillars. Our EBIC images reveal uniform PN junctions conformally constructed on the 3D pillar array. Based on Monte-Carlo simulations and EBIC modeling, we estimate local carrier separation/collection efficiency that reflects the quality of the PN junction. We find the EBIC efficiency of the pillar array increases with the incident electron beam energy, consistent with the EBIC behaviors observed in a high-quality planar PN junction. The magnitude of the EBIC efficiency of our pillar array is about $70 \%$ at $10 \mathrm{kV}$, slightly lower than that of the planar device $\approx \approx 1 \%)$. We suggest that this reduction could be attributed to the unpassivated pillar surface and the unintended recombination centers in the pillar cores introduced during the DRIE processes. Our results support that the depth-dependent EBIC approach is ideally suitable for evaluating PN junctions formed on micro/nanostructured semiconductors with various geometry.

Keywords: EBIC, Electron beam induced current, EBIC efficiency, Radial junction, PN junction, Micropillar array, Solar cells, Local characterization, Scanning electron microscopy
\end{abstract}

\section{Introduction}

PN junctions are fundamental device elements that have been extensively used in various applications, including integrated electronic circuits, optical sensors and detectors, and energy harvesting and conversion systems (Chu et al. 2019; Sengupta et al. 1998; Neudeck 1989). Recent advances in micro/nanofabrication have enabled threedimensional (3D) architectures that offer design flexibility to produce high-performance optoelectronic devices using cost-effective semiconductors (Garnett and Yang 2010; Li 2012; Yoon et al. 2010; Um et al. 2015). These low-quality materials, however, exhibit short minority carrier diffusion lengths $\left(L_{n, p}<10 \mu \mathrm{m}\right)$ due to high concentrations of

\footnotetext{
* Correspondence: heayoung.yoon@utah.edu

'Electrical and Computer Engineering, University of Utah, Salt Lake City, UT 84112, USA

${ }^{2}$ Materials Science and Engineering, University of Utah, Salt Lake City, UT 84112, USA
}

impurities and structural defects (e.g., point defect, vacancy, dislocation, grain boundary), limiting device performance designed in a planar geometry. In contrast, a radial-junction configuration maximizes light absorption along the length of the pillars while extracting minority carriers in the radial direction, effectively decoupling the competing processes. The length of the pillars is sufficiently tall to provide adequate light absorption of the indirect bandgap $\mathrm{Si}$ absorber, but their diameter allows photo-generated carriers to travel a much shorter distance as compared to traditional structures, increasing the carrier extraction efficiency (Kayes et al. 2005). Additionally, the 3D pillar geometry can be further tailored to reduce reflection and increase light-trapping (Garnett and Yang 2010; Kelzenberg et al. 2010). Our previous work demonstrated over twofold higher power conversion efficiencies with radial junction solar cells compared to their planar

\section{Springer Open}



(c) The Author(s). 2020 Open Access This article is licensed under a Creative Commons Attribution 4.0 International License, which permits use, sharing, adaptation, distribution and reproduction in any medium or format, as long as you give appropriate credit to the original author(s) and the source, provide a link to the Creative Commons licence, and indicate if changes were made. The images or other third party material in this article are included in the article's Creative Commons licence, unless indicated otherwise in a credit line to the material. If material is not included in the article's Creative Commons licence and your intended use is not permitted by statutory regulation or exceeds the permitted use, you will need to obtain permission directly from the copyright holder. To view a copy of this licence, visit http://creativecommons.org/licenses/by/4.0/. 
junction counterparts (Yoon et al. 2010; Kendrick et al. 2010; Yoon et al. 2011).

Establishing robust junctions is essential for highperformance PN devices, as it controls the flow of excess carriers in one direction, but not the other, providing rectifying characteristics. Various fabrication methods have been proposed and demonstrated to construct the 3D structures with conformal junction formation using top-down (Dowling et al. 2017; Zeniou et al. 2014; Huang et al. 2011; Han et al. 2014; Qian et al. 2020) or bottom-up approaches (Lew and Redwing 2003; Yoo et al. 2013). Plasma-based deep reactive ion etching (DRIE), also known as the "Bosch process" (Laermer and Schilp 2003), has been widely used for 3D patterning owing to the fast and easy processing with reproducible structures. By repeating a cycle of plasma ion etching and conformal polymer coating, DRIE enables the production of high-aspect-ratio structures on various semiconductor substrates. However, the aggressive etching processes often cause unintended surface damage (e.g., porous surface structures, electrically-active defect centers) (Oehrlein 1989; Wu et al. 2010). An inhomogeneous shallow PN junction created on this (sub)surface may introduce poor rectification and inferior diode characteristics. Much of the research activity has been focused on the optimization of fabrication approaches, whereas there are limited studies on the critical PN junction properties of 3D etched Si pillar arrays.

Electron beam induced current (EBIC) microscopy is a powerful analytical technique for studying local electronic states of semiconductor materials and devices (Zhou et al. 2020; Leamy 1982). EBIC uses a focused electron beam to create excess carriers (i.e., electronhole pairs) near a Schottky or a PN junction. The generated carriers in the quasi-neutral region are diffused in the ambipolar directions. The portion of carriers that reach the junction depends on the recombination rate of their travel path. Subsequently, the local built-in or applied electric field at the junction separates the electronhole pairs, producing an induced current (i.e., EBIC) in the external circuit. EBIC imaging is frequently used to map excess carrier recombination in semiconductors (Teplin et al. 2015). By fitting an EBIC profile that exponentially decays with distance from the junction, a minority carrier diffusion length can be determined (Yakimov 2015). Moreover, recent studies have proposed advanced EBIC simulations and numerical modeling for quantitative analysis of convoluted EBIC signals (Zhou et al. 2020; Haney et al. 2016).

In this work, we report measurements of radial PN junctions formed on Si micropillar arrays based on depthdependent EBIC microscopy. By controlling the beam energy that determines the interaction volume between the injected electron beam and the Si structures, we measure the EBIC characteristics of the pillar surface as well as the pillar cores. Our radial PN junctions consist of $p$-type Si micropillar cores fabricated by DRIE and an $n$-type Si shell formed by phosphorous diffusion. We perform EBIC from the sidewall of the pillars to visualize the conformal PN junction. The junction quality of the micropillar array is determined by extracting a local carrier separation/collection efficiency using simulations and EBIC modeling. We compare the EBIC efficiency of the radial junction to the baseline planar PN junction, providing qualitative and quantitive assessment.

\section{Materials and methods}

\section{Fabrication of Si micropillar arrays}

The radial PN junctions studied in this work consist of $p$-type Si micropillar cores fabricated by DRIE and an $n$ type $\mathrm{Si}$ shell formed by phosphorous gas diffusion. Details of full fabrication steps can be found elsewhere (Yoon et al. 2010, 2011). Briefly, the process was begun by conventional lithography to pattern a $200 \mathrm{~nm}$ thermal oxide layer on Si (p-type; $\rho=0.01 \sim 0.02 \Omega \mathrm{cm}$ ). The patterned $\mathrm{SiO}_{2}$ layer serves as an etch mask in the subsequent anisotropic Si DRIE etching. In DRIE, a cycle of sulfur hexafluoride $\left(\mathrm{SF}_{6}\right)$ etching and octafluorocyclobutane $\left(\mathrm{C}_{4} \mathrm{~F}_{8}\right)$ /oxygen $\left(\mathrm{O}_{2}\right)$ polymer coating (Fig. 1a) was repeated. The $\mathrm{C}_{4} \mathrm{~F}_{8} / \mathrm{O}_{2}$ polymer coating protects the sidewalls, serving to increase the downward anisotropy of the subsequent $\mathrm{SF}_{6}$ etching step and improve verticality of the resulting pillars. Each cycle is composed of (i) $3.5 \mathrm{~s} \mathrm{SF}_{6}$, (ii) $1.5 \mathrm{~s} \mathrm{C}_{4} \mathrm{~F}_{8}+\mathrm{O}_{2}$, and (iii) $1 \mathrm{~s} \mathrm{O}_{2}$ at an $\mathrm{RF}$ power of $1500 \mathrm{~W}$, providing a $\mathrm{Si}$ etch rate of about $2.5 \mu \mathrm{m} / \mathrm{min}$. To remove the sidewall roughness of the pillar arrays, we used several steps of cleaning processes: (i) $\mathrm{O}_{2}$ plasma cleaning $\left(200 \mathrm{sccm} \mathrm{O}_{2}\right.$ flow rate, $30 \mathrm{mTorr}$ chamber pressure, and $1800 \mathrm{~W}$ RF power for $5 \mathrm{~min}$ ), (ii) Piranha cleaning $\left(\mathrm{H}_{2} \mathrm{SO}_{4}: \mathrm{H}_{2} \mathrm{O}_{2}=1: 1\right)$, and (iii) two successive wet oxidation $\left(1000^{\circ} \mathrm{C}\right.$ for $25 \mathrm{~min}$, resulting in $\approx$ $300 \mathrm{~nm}$ thick $\mathrm{SiO}_{2}$ ) and oxide removal processes $(10 \%$ $\mathrm{HF}$ for $1 \mathrm{~min}$ ). To form radial PN junctions, we used gas phase diffusion of a phosphorus oxychloride $\left(\mathrm{POCl}_{3}\right)$ source at $1000^{\circ} \mathrm{C}$ for $13 \mathrm{~min}$. A $300 \mathrm{~nm}$-thick aluminum (Al) metal film was thermally evaporated on the backside of the $p$-type $\mathrm{Si}$ and annealed at $600^{\circ} \mathrm{C}$ for $10 \mathrm{~min}$ in the $\mathrm{N}_{2}$ atmosphere. The frontside contacts to the $n^{+}$diffused layers were formed with indium dots on the four corners of the pillar arrays after a native oxide removal in buffered oxide etchant (BOE 1:50, $30 \mathrm{~s}$ ).

\section{Si planar PN junction controls}

A high-quality Si planar PN device served as a baseline control to evaluate our radial junctions formed on the DRIE-fabricated Si micropillar arrays. We purchased a batch of commercially available $\mathrm{Si}$ solar cells (Solar Made) fabricated with high-quality, single-crystalline $\mathrm{Si}$ 


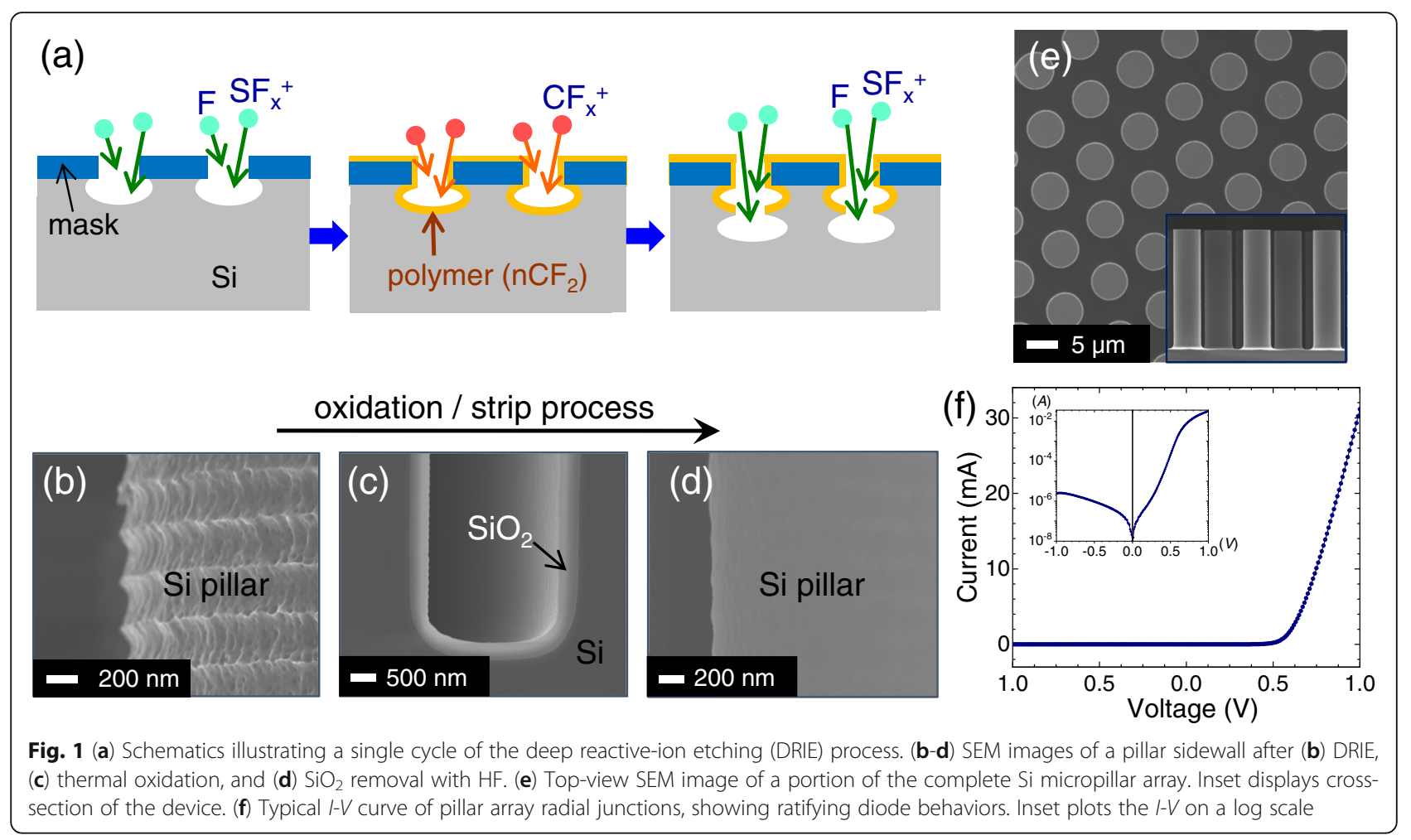

materials. The surface of each device was passivated (e.g., $\mathrm{SiN}$ ) to reduce the surface recombination velocity. Previously, we conducted a cross-sectional EBIC measurement using this sample. The obtained carrier separation/collection efficiency near the planar PN junction was close to $100 \%$, suggesting a robust PN junction of the planar device (Yoon et al. 2014). In this work, we perform EBIC in a depth-dependent configuration.

\section{EBIC characterizations}

EBIC measurements were carried out in a scanning electron microscope (SEM) equipped with a nanomanipulator. This probe arm was used for the placement of an electrical probe on a metal contact of the device, whereas the bottom metal contact was earthed to the SEM stub. The electrical wires were fitted with coaxial electrical feedthrough of the SEM, connecting to an EBIC current amplifier. Once the electrical contacts were made, a series of EBIC/SEM images were acquired at different incident beam energies using a software package.

\section{Monte Carlo simulations (e-beam)}

We used numerical simulations to estimate the twodimensional (2D) profile in $\mathrm{Si}$ at various incident beam energies (CASINO software package: Monte CArlo SImulation of electroN trajectory in sOlids) (Drouin et al. 2007). In each simulation, we used 200,000 electrons at a fixed accelerating voltage in the range of $1 \mathrm{kV}$ to $30 \mathrm{kV}$. The injected electrons were propagated in the
Si matrix via elastic and inelastic scatterings until their energy becomes $50 \mathrm{eV}$ or less. The radius of the normal incident beam was set to $2 \mathrm{~nm}$. A homogeneous Si substrate in a planar geometry was used for the simulation.

\section{Results and discussion}

Figure 1 displays the key fabrication steps to create $\mathrm{Si}$ micropillar arrays. Deep reactive ion etching (DRIE) is a well-established fabrication technique to produce 3D structures by repeating the cycle of a plasma ion etching and a conformal polymer coating (Fig. 1a). These etching/coating processes, however, introduce unintended porous surface structures and electrically-active defect centers. An example of the scalloping profile of the etched structures is shown in Fig. 1b. To remove this structural damage, we used two successive thermal oxidation $\left(1000^{\circ} \mathrm{C}\right.$ for $25 \mathrm{~min}$; $\approx 300 \mathrm{~nm}$ thick $\mathrm{SiO}_{2}$; Fig. 1c) and oxide removal processes (10\% HF for $1 \mathrm{~min}$ ). A representative SEM image in Fig. 1d confirms the dramatically enhanced surface smoothness of the Si micropillars after the rigorous cleaning and oxidation/strip processes. We formed the radial PN junction using gas diffusion of $n$-type phosphorous dopants. An estimated surface doping concentration $\left(N_{d}\right)$ is $\approx 10^{20} \mathrm{~cm}^{-3}$ with a junction depth $\left(x_{j}\right)$ of $\approx 0.3 \mu \mathrm{m}$ (Neudeck 1989). The complete Si micropillars measured $\approx 30 \mu \mathrm{m}$ in height and $\approx 7 \mu \mathrm{m}$ in diameter with a distance between the pillars of approximately $4 \mu \mathrm{m}$ (Fig. 1e). The current-voltage (I-V) curves of the pillar array in Fig. If showed a good diode 
behavior. We estimated the turn-on voltage of $0.63 \mathrm{~V}$, the leakage current of $10 \mathrm{nA}$, and the ideality factor of about 1.7. Comprehensive dark and light $I-V$ characteristics of the radial junctions in various geometrical parameters (e.g., diameter, height, pillar-to-pillar-distance) can be found elsewhere (Yoon et al. 2010, 2011). While extremely informative, $I-V$ curves reflect the overall $\mathrm{PN}$ junction performance and do not capture the local junction properties.

EBIC microscopy allows a direct access to the local $\mathrm{PN}$ junction in $3 \mathrm{D}$ with an adjustable probe size from 10 's $\mathrm{nm}$ to several $\mu \mathrm{m}$, in accordance with the interaction volume between the incident electron beam and the semiconductors. To visualize the local junctions at the level of individual pillars, we carefully cleaved the $\mathrm{Si}$ pillar array using a fine scriber and exposed the crosssection. The sample was mounted on an EBIC holder, where the metal contacts of the emitter (i.e., indium dots on $n^{+}-\mathrm{Si}$ ) and the collector (i.e., $\mathrm{Al}$ on $p$-Si) were connected to the external EBIC circuit. The electron beam was injected from the $n$-Si emitter shell to the PN depletion region and the $p$-Si pillar core. Figure 2 displays an SEM and the corresponding $5 \mathrm{keV}$ (Fig. $2 \mathrm{~b}$ ) and $10 \mathrm{keV}$ (Fig. 2c) EBIC images. Figure 2d shows the overlaid SEM and $5 \mathrm{keV}$ EBIC, indicating a continuous PN junction formed across the 3D geometry. The overall EBIC intensities of the individual pillars at $5 \mathrm{keV}$ and $10 \mathrm{keV}$ are relatively uniform, suggesting the presence of conformal radial junctions along the individual micropillars.

For a quantitative analysis, we extract the EBIC line scans along/across the pillars. The line scan plot across the pillar diameter (Fig. 2e) shows the highest EBIC value near the pillar center $(\approx 80 \mathrm{nA})$ that decreases gradually with the electron beam probe moving away to the perimeter of the pillar $(\approx 50 \mathrm{nA})$. Considering the direction of the incident electron beam to the curved pillar surface, as illustrated in the inset of Fig. 2e, we suggest that this EBIC change is mainly attributed to the shape of the pillar rather than due to inhomogeneous junction properties. With the increase of the backscattered electrons (BSE) and the decrease of the effective electron-hole pair (EHP) generation volume at the curved pillar surface, the reduction of the EBIC magnitude is evident near the pillar perimeter.

Figure $2 \mathrm{f}$ displays the EBIC line scans along the length of the pillars (i.e., axial-direction), showing a relatively constant EBIC value within the pillars at a fixed accelerating voltage. The mean EBIC value of the pillar
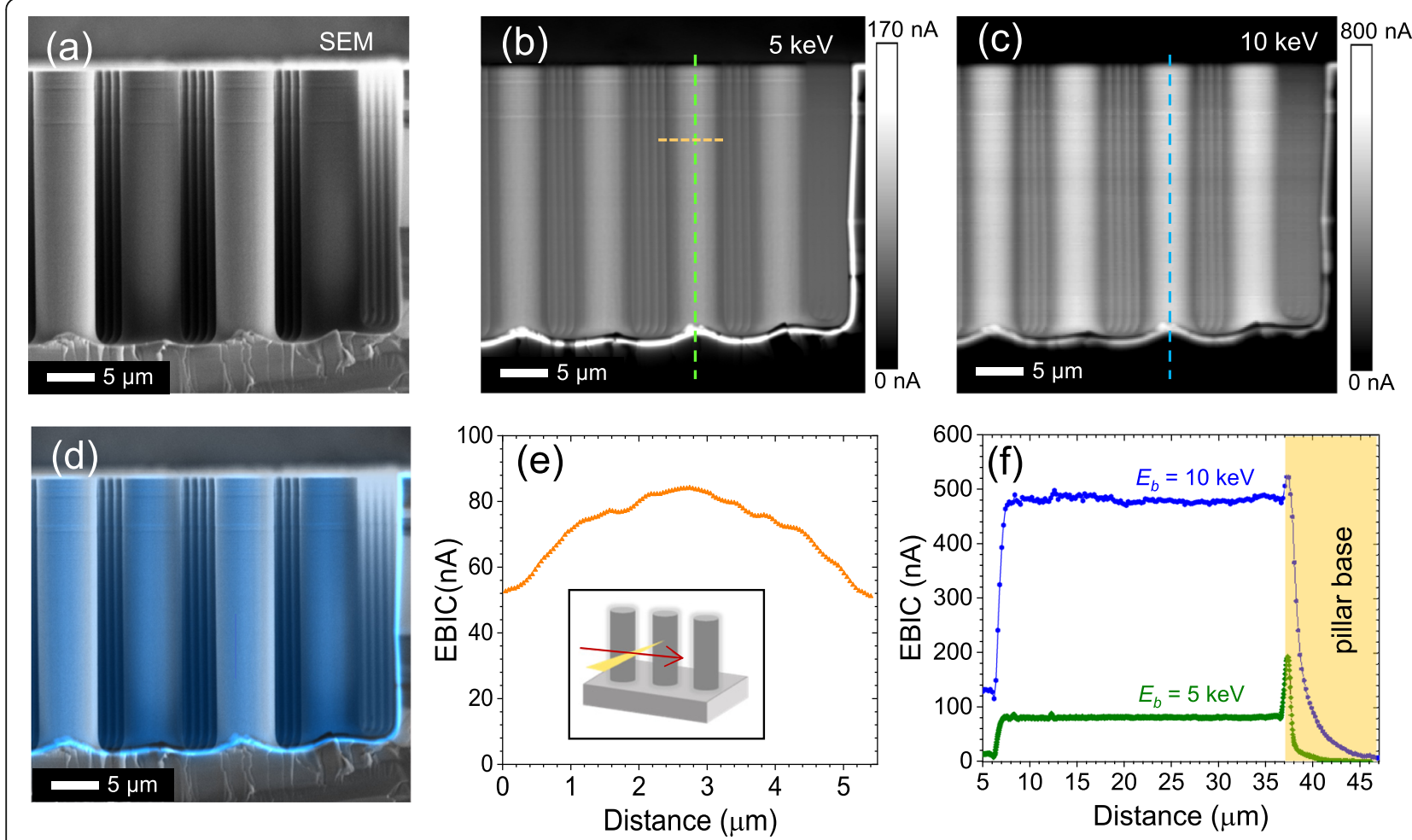

Fig. 2 (a) SEM image of micropillar array and corresponding EBIC (electron beam induced current) maps at (b) 5 keV and (c) $10 \mathrm{keV}$. The relative signal uniformity suggests the presence of conformal radial junctions of individual pillars. (d) Overlay of SEM with 5 keV EBIC image confirms continuous PN junction across device. (e) Extracted EBIC line scan across pillar diameter. (f) EBIC line scans at 5 keV and $10 \mathrm{keV}$ along pillar lengths demonstrate uniform signal magnitude and higher EHP generation at higher energy. Peak EBIC values occur in the pillar base region, where the cross-sectional PN junction is exposed 
increases from $80 \mathrm{nA}$ at $5 \mathrm{keV}$ to $480 \mathrm{nA}$ at $10 \mathrm{keV}$. We observe the highest EBIC values are present in the area of the pillar base, which is associated with the direct local carrier generation within the depletion region. When the electron beam is directly injected to the crosssectional PN junction (i.e., mechanically cleaved junction area), the generated EHPs are separated quickly without diffusion owing to the built-in electric field (C. J. Wu and Wittry 1978; Yakimov 2015). In contrast, the electron beam irradiated on the pillar sidewalls generates the EHPs in the depletion region as well as the chargeneutral regions (i.e., $n$-Si shell, $p$-Si pillar core). The excess carriers must travel to the junction (i.e., ambipolar diffusion) before they are separated and collected in EBIC. Since the $n$-Si emitter layer $\left(N_{d} \approx 10^{20} \mathrm{~cm}^{-3}, x_{j} \approx\right.$ $300 \mathrm{~nm}$ ) of our pillars is conductive, yet highly defective, the EHPs generated in this region tend to be recombined, decreasing overall EBIC values as compared to the direct electron beam injection at the cross-sectional $\mathrm{PN}$ junction. As the EHP generation volume increases with the accelerating voltage (i.e., $5 \mathrm{keV}$ to $10 \mathrm{keV}$ ), the portion of the EHPs generated in the $n$-Si decreases, resulting in a comparable EBIC value of the pillar and near the base.

To assess the local junction quality of the micropillar array, we collected the baseline EBIC characteristics of a commercial planar device (Solar Made). This planar PN junction $\left(n^{+}-p\right)$ was built on a high-purity single crystalline Si substrate, and it showed a carrier collection efficiency close to $100 \%$ in the depletion region obtained in a normal collector EBIC configuration (Yoon et al. 2014). Figure 3 displays a representative SEM image of the planar PN device and the corresponding EBIC maps collected at $5 \mathrm{keV}$, $10 \mathrm{keV}$, and $20 \mathrm{keV}$. The large dark area of the EBIC images is associated with the metal contact, highlighted in yellow in the SEM image (Fig. 3a). The injected electron beam $(1 \mathrm{keV} \sim 30 \mathrm{keV})$ does not penetrate this thick metal layer (a few $\mathrm{mm}$ thick $\mathrm{Ag}$ paste), producing negligible EBIC signals (Fig. 3b-d). The dark speckles in the $5 \mathrm{keV}$ EBIC image are likely attributed to thin organic residue or dust particles on the sample, of which EBIC contribution becomes insignificant with the higher beam energies $(>10 \mathrm{keV})$. Qualitatively, the bright contrast increases with a higher $\mathrm{keV}$, showing similar behaviors as those observed with the pillar array radial junction (Fig. 2).

Figure $3 \mathrm{f}$ through g show the representative line scans extracted from the EBIC images (Fig. 3b-d). A relatively constant EBIC was observed in the device area, a stack of $p$-Si collector, $n$-Si emitter, and $\mathrm{SiN}$ passivation layer. A notable current fluctuation near the metal contact is mainly attributed to the spread of the metal paste. By aligning the line scans, we find that a low $\mathrm{keV}$ EBIC is much more sensitive to the surface features than higher $\mathrm{keV}$. For instance, two distinct peaks observed in the 5 $\mathrm{keV}$ EBIC line scan (marked with a green box) conform to the sample topography shown in SEM (Fig. 3a). This feature becomes less distinguishable with increasing incident beam energy, as the electron beam penetrates deep in the sample with a larger EHP generation volume. We used the EBIC images from $5 \mathrm{keV}$ to $30 \mathrm{keV}$ and calculated mean EBIC values for the Si area, shown in Fig. 3e. The increase of EBIC with a higher $\mathrm{keV}$ is evident in the line scans. The average EBIC value increases from 127 $\mathrm{nA}$ at $5 \mathrm{kV}$ to $3.55 \mu \mathrm{A}$ at $30 \mathrm{kV}$, increasing over one order of magnitude. Interestingly, the EBIC values observed in the planar junction are slightly higher than those in the radial junction in Fig. 2: $127 \mathrm{nA}$ (vs. $83 \mathrm{nA}$ of the radial junction) at $5 \mathrm{keV}, 574 \mathrm{nA}$ (vs. $492 \mathrm{nA}$ of the radial junction) at $10 \mathrm{keV}$.

The experimental results qualitatively suggest that EBIC magnitude near the PN junctions is strongly influenced by the EHP generation by the incident electron beam and the local carrier separation/collection properties. To gain a deeper understanding of the local radial junction characteristics, we estimate the carrier generation profile using Monte Carlo simulations and calculate the local carrier collection efficiency for the planar and the radial junctions. Figure 4a (top) displays an example of the simulated electron trajectories, where a ray of $5 \mathrm{keV}$ electron beam is irradiated onto a $\mathrm{Si}$ substrate. The blue lines represent the collision events of the primary electrons with $\mathrm{Si}$ until they lose their initial energy (i.e., $5000 \mathrm{~V}$ in this example) to $50 \mathrm{~V}$ or lower. The red lines represent the paths of the backscattered electrons. A corresponding energy contour plot is shown in the bottom image. For instance, the $95 \%$ contour represents the sample area where the injected primary electrons have lost $95 \%$ of their initial energy. Figure $4 \mathrm{~b}$ plots the estimated interaction bulb size at different accelerating voltages $(1 \mathrm{kV} \sim 30 \mathrm{kV})$. The overall ratio of depth to diameter (depth/diameter) is comparable for higher lost-energy contours $(>75 \%)$, yet slightly higher for low energy contours $(<50 \%)$, indicating a pearshape of the interaction bulb. The calculated bulb size at 1 $\mathrm{keV}$ is approximately $19 \mathrm{~nm}$, inferring that the spatial resolution of the EBIC image for flat Si devices can be achieved as high as $<20 \mathrm{~nm}$. The inset of Fig. $4 \mathrm{~b}$ shows the increase of the penetration depth with the beam energy, which was extracted from the 95\% energy contour of each simulation. The numerical fit overlaid on the datasets confirms the bulb size is proportional to $E_{b}^{1.78}$ ( $E_{b}$ is the beam energy), showing an excellent agreement with the analytical prediction of $\approx$ $E_{b}^{1.7}$ by Wittry et al. (Wittry and Kyser 1967). By controlling the incident beam energy, the size of the EHP generation bulb can be tunable from 10's nm to several $\mu \mathrm{m}$, offering versatility to study local carrier dynamics in optoelectronic semiconductor materials and devices. 



Fig. 3 (a) SEM image of planar PN device with metal contact highlighted in yellow. [center top] Schematic of EBIC measurement. [center bottom] Slightly tilted SEM image of the sample, illustrating surface roughness. Corresponding EBIC scans of the planar device at (b) $5 \mathrm{keV}$, (c) $10 \mathrm{keV}$, and (d) $20 \mathrm{keV}$ showing higher contrast and reduced spatial resolution with higher energy. (e) Increasing mean EBIC current calculated for Si line scans. (f-h) Plots of EBIC current along line scans shown with green lines in (b-d): (f) $5 \mathrm{keV}$, (g) $10 \mathrm{keV}$, (h) $20 \mathrm{keV}$. The green box highlights the representative sample topography. The two distinct features at $5 \mathrm{keV}$ become broad and indistinguishable with higher beam energies

Based on the Monte-Carlo simulations and EBIC modeling (Leamy 1982; Haney et al. 2016; Yakimov 2015), we estimated the local carrier separation/carrier efficiency of the radial junction and compared it to planar PN controls. The EBIC collection efficiency $\left(\eta_{E B I C}\right)$ is defined as the ratio of the measured current $\left(I_{E B I C}\right)$ to the EHP generation rate $(\beta)$. Here, $e$ is the unit charge $\left(1.6 \times 10^{-19} \mathrm{C}\right)$.

$$
\eta(E B I C)=\frac{I_{E B I C}}{e \cdot \beta}
$$

The generation rate, which is the total number of EHPs created by the injected electron beam, can be calculated as below.

$$
\beta=\frac{I_{b} \cdot E_{b} \cdot \alpha}{e \cdot E_{E H P}}
$$

$E_{b}$ is the incident electron beam energy, $\alpha$ is the fraction of beam energy absorbed inside the material (i.e., $\mathrm{Si}$ in our case), and $E_{E H P}$ is the average energy to create an electron-hole pair. The $I_{b}$ of our SEM was measured in the range of $250 \mathrm{pA}\left(E_{b}=5 \mathrm{kV}\right)$ to $300 \mathrm{pA}\left(E_{b}=20 \mathrm{kV}\right)$. We calculated the magnitude of $\alpha$ using the backscattered coefficient obtained from the Monte Carlo simulation (e.g., 0.152 at $5 \mathrm{keV}, 0.142$ at $20 \mathrm{keV}$ ). The $E_{E H P}$ was estimated using an empirical relation of $E_{E H P}=2.596$ $E_{g}+0.174$ (Kobayashi et al. 1972), giving $E_{E H P} \approx 3.621$ $\mathrm{eV}$ for $\mathrm{Si}\left(E_{g}=1.12 \mathrm{eV}\right)$. The EBIC currents $\left(I_{b}\right)$ extracted from the line scans in Figs. 2 and 3 were used for the pillar array and the planar device, respectively. 
(a)
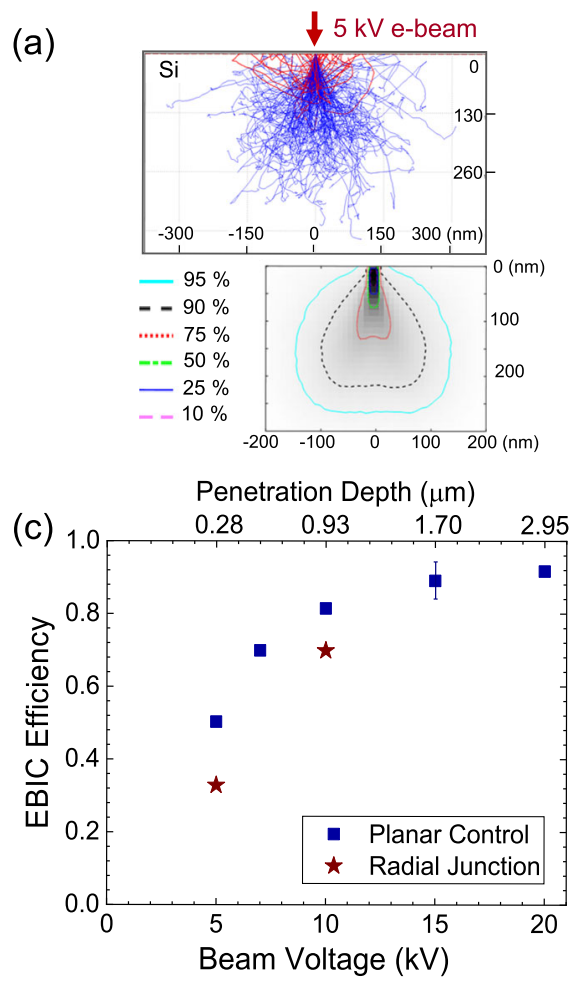
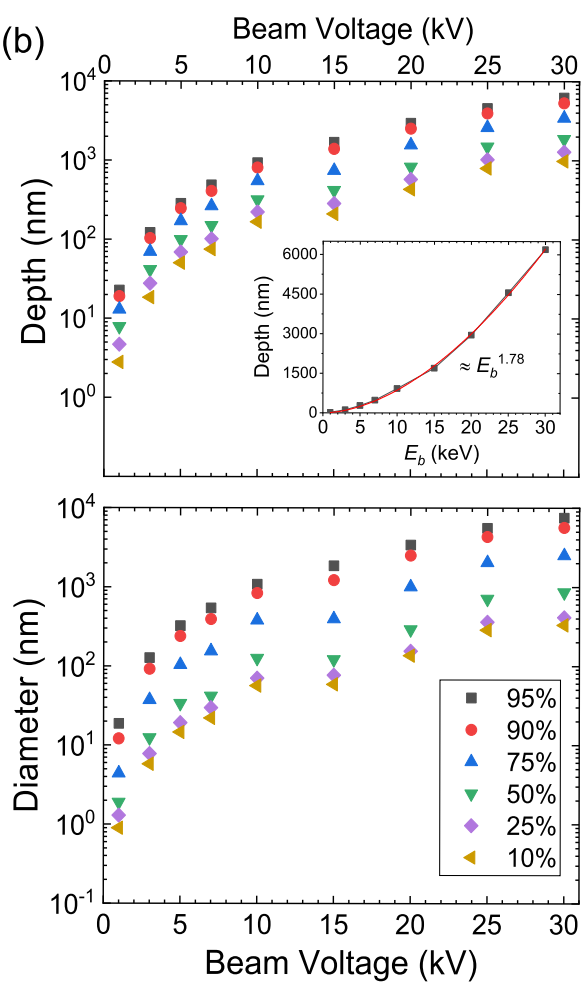

Fig. 4 (a) Simulated electron paths for a ray of $5 \mathrm{kV}$ electron beam in Si (200,000 electrons). Blue lines represent collision events of electrons with Si. Red lines represent backscattered electrons. (a, bottom) Energy contour plot from $5 \mathrm{kV}$ simulation with percent energy loss contours. (b) Simulated interaction bulb size at various accelerating voltages: [top] maximum depth and [bottom] maximum diameter. Inset shows the penetration depth with beam energy (95\% energy contour) and corresponding curve fit. (c) Estimated EBIC efficiency of the radial junction compared to the planar PN junction control

We note that a typical uncertainty in our EBIC measurement and analysis is about $10 \%$ associated with the fluctuations of the baseline e-beam current $\left(I_{b}\right)$ and the signal-to-noise ratio of the EBIC preamplifier. Also, the parameters extracted from the Monte Carlo simulations (e.g., backscattered coefficient, mean EHP generation rate $(\beta)$, empirical parameter $\left(E_{E H P}\right)$ to generate EHPs) contribute to the uncertainty.

Finally, we plot the resulting EBIC efficiency of the devices at different incident beam energies in Fig. 4c. The EBIC efficiency increases with the incident beam energy, reaching close to unity at $E_{b}>15 \mathrm{keV}$ for the planar PN junction device. A similar trend was observed for the radial junction of the pillar array, yet the overall EBIC efficiency is slightly lower than that of the planar device (about 10\%). In both cases, EBIC was measured in the depth-dependent configuration. The injected electrons travel from the highly-doped emitter (a few $100 \mathrm{~nm}$ thick) to the depletion region $(<1 \mu \mathrm{m})$ and the $p$-Si collector, generating the EHPs in three different layers. The low EBIC efficiency at $5 \mathrm{keV}$ (50\% for the planar device; $30 \%$ for the pillar array) is likely attributed to the EHP production in the highly-doped emitter region. Our Monte Carlo simulation shows an interaction bulb size of $(300 \mathrm{~nm})^{3}$ at $5 \mathrm{keV}$, suggesting that most EHPs were produced in the highly-defective (i.e., high-density of recombination centers) emitter region that promotes excess carrier recombination. At higher $\mathrm{keV}$, most EHPs are generated in the strong built-in electric field region and the collector, increasing the EBIC efficiency. Our observation indicates that the surface damage introduced on the pillars by DRIE could be effectively removed by rigorous cleaning and oxidation/strip processes. The magnitude of the EBIC efficiency of our pillar array is about $70 \%$ at $10 \mathrm{kV}$, slightly lower than that of the planar device $(\approx 81 \%)$. We speculate that a slightly higher EBIC efficiency for the planar junction is associated with the surface passivation (e.g., $\mathrm{SiN}$ ) that decreases the surface recombination of EHPs. Detailed EBIC studies of the surface passivation based on 3D continuity equations together with Poisson equations (Yakimov 2015; Haney et al. 2016; Zhou et al. 2020) will provide additional insight on the excess carrier dynamics and general guidance to improve their device performance.

\section{Summary and conclusions}

In summary, we have examined the radial junction characteristics of $\mathrm{Si}$ micropillar arrays using depth- 
dependent EBIC microscopy. The EBIC images collected from the sidewall of the pillars confirm the uniform PN junction conformally constructed on the 3D pillar array. We find the EBIC efficiency of the pillar array increases with the injected electron-beam voltage, consistent with the EBIC behaviors observed in a high-quality planar PN junction. The magnitude of the EBIC efficiency of our pillar array is about $70 \%$ at $10 \mathrm{kV}$, slightly lower than that of the planar device $(\approx 81 \%)$. We suggest that this reduction could be attributed to the unpassivated pillar surface or the low material quality of the pillar core. Our results support that the depth-dependent EBIC approach is ideally suitable for evaluating $3 \mathrm{D}$ conformal PN junctions formed on micro/nanostructures with various geometries.

\section{Abbreviations}

2D: Two-dimensional; 3D: Three-dimensional; BSE: Backscattered electron; CASINO: Monte-CArlo SImulation of electroN trajectory in sOlids; DRIE: Deep reactive-ion etching; EBIC: Electron-beam induced current; EHP: Electron-hole pair; RF: Radio frequency; SEM: Scanning electron microscopy

\section{Acknowledgements}

The authors acknowledge the support from B. Baker, D. Magginetti, and S. Pritchett for the development of device fabrication. The radial junction processes and the EBIC data acquisition were performed at Penn State University (University Park, PA, USA) and the National Institute of Standards and Technology (Gaithersburg, MD, USA). We thank Y. Yuwen, T. Mayer, P. Haney, and N. Zhitenev for valuable discussions.

\section{Authors' contributions}

KMP and HPY have contributed to sample preparation, data acquisition, data analysis, and manuscript writing. HPY supervised the overall project. All authors read and approved the final manuscript.

\section{Funding}

This research was supported by a University of Utah Seed Grant and New Faculty Start-up Funds. We acknowledge support by the USTAR shared facilities at the University of Utah, in part, by the MRSEC Program of NSF under Award No. DMR-1121252.

\section{Availability of data and materials}

The datasets used in this study are available from the corresponding author on reasonable request.

\section{Competing interests}

The authors declare that they have no competing interests.

Received: 15 July 2020 Accepted: 24 August 2020

Published online: 03 September 2020

\section{References}

Y.H. Chu, C.Q. Qian, P. Chahal, C.Y. Cao, Printed diodes: Materials processing, fabrication, and applications. Adv. Sci. 6(6), 1801653 (2019). https://doi.org/10. 1002/advs.201801653

K.M. Dowling, E.H. Ransom, D.G. Senesky, Profile evolution of high aspect ratio silicon carbide trenches by inductive coupled plasma etching. J. Microelectromech. Syst. 26(1), 135-142 (2017). https://doi.org/10.1109/ Jmems.2016.2621131

D. Drouin, A.R. Couture, D. Joly, X. Tastet, V. Aimez, R. Gauvin, CASINO V2.42 - a fast and easy-to-use modeling tool for scanning electron microscopy and microanalysis users. Scanning 29(3), 92-101 (2007). https://doi.org/10.1002/ sca. 20000

E. Garnett, P.D. Yang, Light trapping in silicon nanowire solar cells. Nano Lett. 10(3), 1082-1087 (2010). https://doi.org/10.1021/nl100161z
H. Han, Z.P. Huang, W. Lee, Metal-assisted chemical etching of silicon and nanotechnology applications. Nano Today 9(3), 271-304 (2014). https://doi. org/10.1016/j.nantod.2014.04.013

P.M. Haney, H.P. Yoon, B. Gaury, N.B. Zhitenev, Depletion region surface effects in electron beam induced current measurements. J. Appl. Phys. 120(9), 095702 (2016). https://doi.org/10.1063/1.4962016

Z.P. Huang, N. Geyer, P. Werner, J. de Boor, U. Gosele, Metal-assisted chemical etching of silicon: A review. Adv. Mater. 23(2), 285-308 (2011). https://doi. org/10.1002/adma.201001784

B.M. Kayes, H.A. Atwater, N.S. Lewis, Comparison of the device physics principles of planar and radial p-n junction nanorod solar cells. J. Appl. Phys. 97(11), 114302 (2005). https://doi.org/10.1063/1.1901835

M.D. Kelzenberg, S.W. Boettcher, J.A. Petykiewicz, D.B. Turner-Evans, M.C. Putnam, E.L. Warren, et al., Enhanced absorption and carrier collection in Si wire arrays for photovoltaic applications. Nat. Mater. 9(3), 239 (2010) <Go to ISI>://WOS: 000274700900021

C.E. Kendrick, H.P. Yoon, Y.A. Yuwen, G.D. Barber, H.T. Shen, T.E. Mallouk, et al., Radial junction silicon wire array solar cells fabricated by gold-catalyzed vapor-liquid-solid growth. Appl. Phys. Lett. 97(14), 143108 (2010). https://doi. org/10.1063/1.3496044

T. Kobayashi, M. Koyama, T. Sugita, S. Takayanagi, Performance of GaAs surfacebarrier detectors made from high-purity gallium-arsenide. IEEE Trans. Nucl. Sci. 19(3), 324-32+ (1972). https://doi.org/10.1109/Tns.1972.4326745

Laermer, F., \& Schilp, A. (2003). Method of anisotropic etching of silicon. Patent US6531068 (US)

H.J. Leamy, Charge collection scanning electron-microscopy. J. Appl. Phys. 53(6), R51-R80 (1982). https://doi.org/10.1063/1.331667

K.K. Lew, J.M. Redwing, Growth characteristics of silicon nanowires synthesized by vapor-liquid-solid growth in nanoporous alumina templates. J. Cryst. Growth 254(1-2), 14-22 (2003). https://doi.org/10.1016/S0022-0248(03)01146-1

X.L. Li, Metal assisted chemical etching for high aspect ratio nanostructures: A review of characteristics and applications in photovoltaics. Curr. Opin. Solid State Mater. Sci. 16(2), 71-81 (2012). https://doi.org/10.1016/j.cossms.2011.11.002

G.W. Neudeck, The PN Junction Diode (Addison-Wesley, Reading, 1989)

G.S. Oehrlein, Dry etching damage of silicon - a review. Mater. Sci. Eng. B Solid State Mater. Adv. Technol. 4(1-4), 441-450 (1989). https://doi.org/10.1016/ 0921-5107(89)90284-5

Qian, Y., Magginetti, D. J., Jeon, S., Yoon, Y., Olsen, T. L., Wang, M., et al. (2020). Heterogeneous optoelectronic characteristics of Si micropillar arrays fabricated by metal-assisted chemical etching. https:/ui.adsabs.harvard.edu/ abs/2020arXiv200616308Q. Accessed 1 June 2020

D.L. Sengupta, T.K. Sarkar, D. Sen, Centennial of the semiconductor diode detector. Proc. IEEE 86(1), 235-243 (1998). https://doi.org/10.1109/5.658775

C.W. Teplin, S. Grover, A. Chitu, A. Limanov, M. Chahal, J. Im, et al., Comparison of thin epitaxial film silicon photovoltaics fabricated on monocrystalline and polycrystalline seed layers on glass. Prog. Photovolt. 23(7), 909-917 (2015). https://doi.org/10.1002/pip.2505

H.D. Um, N. Kim, K. Lee, I. Hwang, J.H. Seo, Y.J. Yu, et al., Versatile control of metal-assisted chemical etching for vertical silicon microwire arrays and their photovoltaic applications. Sci. Rep. 5, 11277 (2015). https://doi.org/10.1038/ srep 11277

D.B. Wittry, D.F. Kyser, Measurement of diffusion lengths in direct-gap semiconductors by electron-beam excitation. J. Appl. Phys. 38(1), 375 (1967). https://doi.org/10.1063/1.1708984

B.Q. Wu, A. Kumar, S. Pamarthy, High aspect ratio silicon etch: A review. J. Appl. Phys. 108(5), 051101 (2010). https://doi.org/10.1063/1.3474652

C.J. Wu, D.B. Wittry, Investigation of minority-carrier diffusion lengths by electronbombardment of Schottky barriers. J. Appl. Phys. 49(5), 2827-2836 (1978). https://doi.org/10.1063/1.325163

E.B. Yakimov, What is the real value of diffusion length in GaN? J. Alloys Compd. 627, 344-351 (2015). https://doi.org/10.1016/j.jallcom.2014.11.229

J. Yoo, S.A. Dayeh, W. Tang, S.T. Picraux, Epitaxial growth of radial Si p-i-n junctions for photovoltaic applications. Appl. Phys. Lett. 102(9), 093113 (2013). https://doi.org/10.1063/1.4794541

H.P. Yoon, P.M. Haney, J. Schumacher, K. Siebein, Y. Yoon, N.B. Zhitenev, Effects of focused-ion-beam processing on local electrical measurements of inorganic solar cells. Microsc. Microanal. 20(S3), 544-545 (2014). https://doi.org/10.1017/ S1431927614004449

H.P. Yoon, Y.A. Yuwen, C.E. Kendrick, G.D. Barber, N.J. Podraza, J.M. Redwing, et al., Enhanced conversion efficiencies for pillar array solar cells fabricated from 
crystalline silicon with short minority carrier diffusion lengths. Appl. Phys. Lett. 96(21), 213503 (2010). https://doi.org/10.1063/1.3432449

H.P. Yoon, Y.A. Yuwen, H. Shen, N.J. Podraza, T.E. Mallouk, E.C. Dickey, et al., in 37th IEEE Photovoltaic Specialists Conference. Parametric study of micropillar array solar cells (2011), pp. 000303-000306. https://doi.org/10.1109/PVSC. 2011.6185905

A. Zeniou, K. Ellinas, A. Olziersky, E. Gogolides, Ultra-high aspect ratio Si nanowires fabricated with plasma etching: Plasma processing, mechanical stability analysis against adhesion and capillary forces and oleophobicity. Nanotechnology 25(3), 035302 (2014). https://doi.org/10.1088/0957-4484/25/ 3/035302

R.N. Zhou, M.Z. Yu, D. Tweddle, P. Hamer, D. Chen, B. Hallam, et al., Understanding and optimizing EBIC pn-junction characterization from modeling insights. J. Appl. Phys. 127(2), 024502 (2020). https://doi.org/10. $1063 / 1.5139894$

\section{Publisher's Note}

Springer Nature remains neutral with regard to jurisdictional claims in published maps and institutional affiliations.

\section{Submit your manuscript to a SpringerOpen ${ }^{\circ}$ journal and benefit from:}

- Convenient online submission

- Rigorous peer review

- Open access: articles freely available online

- High visibility within the field

- Retaining the copyright to your article

Submit your next manuscript at $\boldsymbol{\nabla}$ springeropen.com 